\title{
Ileal immune tonus is a prognosis marker of proximal colon cancer in mice and patients
}

\author{
Marion Picard ${ }^{1,2,3,4} \cdot$ Satoru Yonekura $\mathbb{D}^{1,2,5} \cdot$ Karolina Slowicka $^{6,7} \cdot$ loanna Petta ${ }^{6,8} \cdot$ Conrad Rauber $^{1,2,5}$. \\ Bertrand Routy ${ }^{1,2,5}$. Corentin Richard ${ }^{9,10}$ - Valerio lebba ${ }^{11}$ - Maryam Tidjani Alou ${ }^{1,2}$ • Sonia Becharef ${ }^{1,2} \cdot$ Pierre Ly $^{1,2}$. \\ Eugenie Pizzato $^{1,2} \cdot$ Christian H. K. Lehmann ${ }^{12}$. Lukas Amon ${ }^{12}$. Christophe Klein $\mathbb{D D}^{13}$ - Paule Opolon ${ }^{14}$. \\ Ivo Gomperts Boneca $\mathbb{1}^{3,4,15}$ - Jean-Yves Scoazec ${ }^{14}$ - Antoine Hollebecque ${ }^{16}$. David Malka ${ }^{16}$. \\ François Ghiringhelli $\mathbb{D}^{9,10}$ - Diana Dudziak ${ }^{12}$ - Geert Berx ${ }^{7,17} \cdot$ Lars Vereecke $\mathbb{1}^{6,8} \cdot$ Geert van Loo $\mathbb{D}^{6,7}$. \\ Guido Kroemer ${ }^{13,18,19,20,21} \cdot$ Laurence Zitvogel (i) $^{1,2,5,21,22} \cdot$ Maria Paula Roberti ${ }^{1,2,22,23}$
}

Received: 6 August 2020 / Revised: 11 November 2020 / Accepted: 13 November 2020 / Published online: 1 December 2020

(c) The Author(s) 2020. This article is published with open access

\begin{abstract}
Ileal epithelial cell apoptosis and the local microbiota modulate the effects of oxaliplatin against proximal colon cancer by modulating tumor immunosurveillance. Here, we identified an ileal immune profile associated with the prognosis of colon cancer and responses to chemotherapy. The whole immune ileal transcriptome was upregulated in poor-prognosis patients with proximal colon cancer, while the colonic immunity of healthy and neoplastic areas was downregulated (except for the Th17 fingerprint) in such patients. Similar observations were made across experimental models of implanted and spontaneous murine colon cancer, showing a relationship between carcinogenesis and ileal inflammation. Conversely, oxaliplatin-based chemotherapy could restore a favorable, attenuated ileal immune fingerprint in responders. These results suggest that chemotherapy inversely shapes the immune profile of the ileum-tumor axis, influencing clinical outcome.
\end{abstract}

\section{Introduction}

The field of cancer treatment has seen major advancements over the last few years with the approval of immune checkpoint inhibitors (ICI) for multiple indications. However, ICIs do not benefit all patients; there are still major challenges to overcome primary or secondary resistance against immunotherapy. For colorectal cancer (CRC),

These authors contributed equally: Marion Picard, Satoru Yonekura

These authors jointly supervised this work: Laurence Zitvogel, Maria Paula Roberti

Edited by H.-U. Simon

Supplementary information The online version of this article (https:// doi.org/10.1038/s41418-020-00684-w) contains supplementary material, which is available to authorized users.

Maria Paula Roberti

mariapaula.roberti@nct-heidelberg.de

Extended author information available on the last page of the article microsatellite stability (MSS) is resistant to ICI and represents the vast majority of CRC [1]. Combination chemotherapy based on the use of 5-fluorouracil (5-FU) plus oxaliplatin (OXA) (FOLFOX) is routinely used as first-line treatment for advanced CRC [2], although with no curative intention. Therefore, it is important to improve treatment efficacy by combining standard chemotherapy with ICI. To do so, the identification of new therapeutic options and associated biomarkers will help selecting patients for clinical trials and evaluating therapeutic responses.

Recently, the interaction between the gut-associated immune system (GALT) and the immune tumor microenvironment (TME) has been recognized. Evidence from preclinical models and patients unraveled the gut microbiota and local immunity as a crucial crossroad in the modulation of responses to cancer therapies (reviewed in ref. [3]). The gut immune response is the result of a fine-tuned homeostatic equilibrium between immunity to pathogenic antigens and tolerance to nonpathogenic ones. Interactions between the microbiota, the intestinal epithelial barrier, and GALT continuously shape the function of the gut immune system [4]. Chemotherapy can affect the integrity of the gut 
epithelial barrier, thus altering the outcome of the local and systemic immune response.

Our group provided mechanistic insights into how the microbiota and apoptosis of intestinal epithelial cell (IEC) in the ileum elicit an immune response against proximal colon cancer (pCC) in the context of OXA-based chemotherapy [5]. The local priming of immune cells in the mesenteric lymph nodes $(\mathrm{mLN})$ culminated in the enrichment of $\mathrm{CD}^{+} \mathrm{T}$ cells, $\mathrm{CD}^{+}$cytotoxic $\mathrm{T}$ lymphocytes (CTL), and T follicular helper (Tfh) in the TME, indicating a favorable immune contexture [6, 7] associated with good prognosis and treatment response [8]. Whether and how the local mucosal immune profile was impacted remained unexplored. In the present study, we describe ileal mucosal immune changes in pCC patients and their association to clinical outcome. Furthermore, we demonstrate cause-effect relationships among the different factors involved in shaping ileal immunity using relevant experimental mouse models of colon cancer.

We reveal that favorable tumor immunity and clinical outcome are associated with a relative loss of immune markers in the ileum. Using spontaneous and transplantable colon cancer mouse models, we identified functional links among tumor, intestinal microbes, and chemotherapy impacting on ileal immunity, resulting in apparently opposite changes between ileal inflammation and tumor immunosurveillance. We believe that these findings are important for the future development of new treatment combinations against colon cancers.

\section{Results}

\section{Ileal immune parameters dictate pCC patients' prognosis}

We recently provided insights into how ileal IEC death and the local microbiota elicit a systemic immune response against pCC during OXA-based chemotherapy [5]. Indeed, we showed that Tfh primed in the mLNs post OXA accumulates in colonic tumor beds instead of being redirected toward the chemotherapy-inflamed ileum. In these pCC patients, we analyzed the ileal immune tonus and its impact on patient survival.

Right hemicolectomy is the standard surgical treatment for malignant neoplasms of the right colon, which involves the resection of the tumor along with nonmalignant tissues, including the terminal ileum. This procedure allowed us to concomitantly monitor the ileal and tumoral immune parameters, the ileal and colonic microbiota lining healthy tissues, and pCC patient prognosis.

Immunofluorescence-based staining of T-cell subsets in the ilea and tumor beds revealed an inverse correlation between $\mathrm{CD}^{+}$and $\mathrm{CD}^{+}{ }^{+} \mathrm{T}$ lymphocytes within the ileal epithelium (EP) or lamina propria (LP), and $\mathrm{CD} 8^{+}$tumorinfiltrating lymphocytes (TILs) in the invasive margins of the resected pCC (Fig. 1A, B). Hence, cancer T-cell infiltration (a surrogate marker of prognosis in CRC) [9] is particularly dense in patients whose ilea contain few T cells. Notably, ileal LP Tfh cells exhibited robust anticorrelations with TILs, including tumor-infiltrating Tfh cells (Fig. 1B). We next evaluated $\mathrm{T}$ lymphocyte-related gene expression patterns, focusing on a custom panel comprising 15 transcription factors and cytokine genes, by means of quantitative real-time polymerase chain reaction (RT-qPCR) performed on the terminal ileum and proximal colon segments removed during surgery. This RT-qPCR reaction was validated by protein expression for selected gene products, such as for the aryl hydrocarbon receptor (AHR) for which gene and protein expressions markedly correlated among each other (Supplementary Fig. S1A, B). Nonsupervised hierarchical clustering of immune gene products yielded two opposite clusters of patient samples, based on the geodistribution of gene expression, i.e., cluster 1 in which the mRNAs were scarcely expressed in the ileum but highly expressed in the colon, and cluster 2 in which mRNAs exhibited an opposite pattern with high expression in the ileum and low expression in the colon (Fig. 1C, D). Indeed, CD3E, TBX21, FOXP3, IL10, GATA3, and IL23A were more expressed in the colon while being less expressed in the ileum in cluster 1 compared with cluster 2 (Supplementary Fig. S2, right and left panels). Importantly, these gene expression profiles had a clinical relevance in thus far that cluster 1 patients exhibited a prolonged time to treatment failure (progression or cancer-related death) than those individuals whose ileal signature fell into cluster 2 (Fig. 1E, clinical characteristics of the patients are detailed in Table S1, adjusted $P$ values in Table S2). Importantly, ileal immune gene expression was independent of the disease stage, and ileal immune tonus even predicted survival of stage IV pCC metastatic patients (Fig. 1F).

Altogether, we conclude that the immune tonus of the ileal mucosa correlates with colon cancer immunosurveillance, as well as with patient survival.

\section{Colon cancer promotes high ileal immune gene expression}

Although the ileal immune tonus was independent of tumor stage (Fig. 1C, F), genes coding for $C D 4$, the Tfhrelevant transcription factor $B C L 6$, the $\mathrm{T}$ helper 1-relevant transcription factor $T B X 21$, and $A H R$ were significantly ( $>2$-fold) increased in the ileum (Fig. 2A, left panel) but not in the colon (Fig. 2A, right panel) in more advanced pCC stages (III/IV) compared with early stages of colon carcinogenesis. 
A.

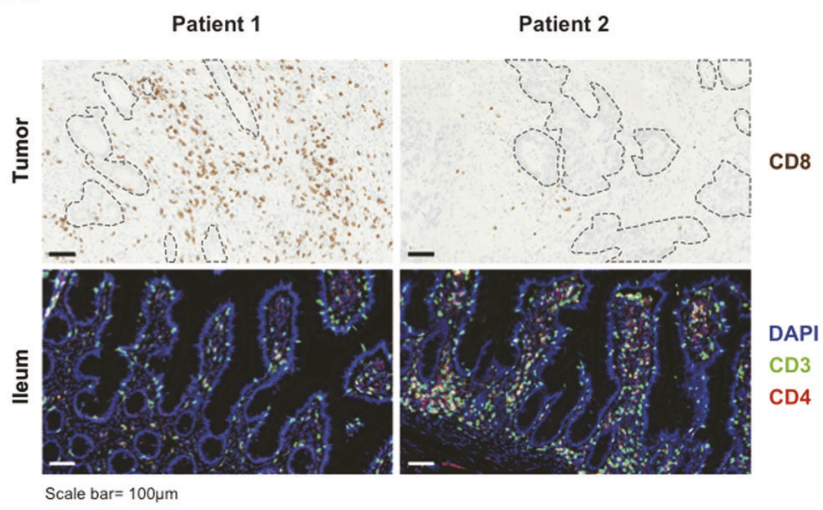

C.

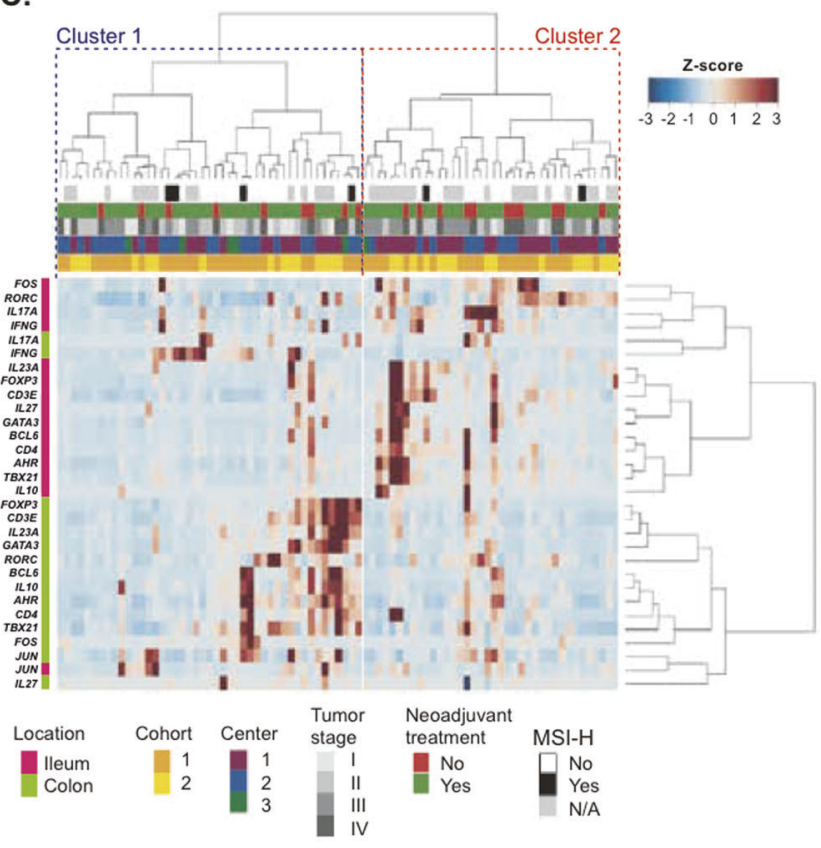

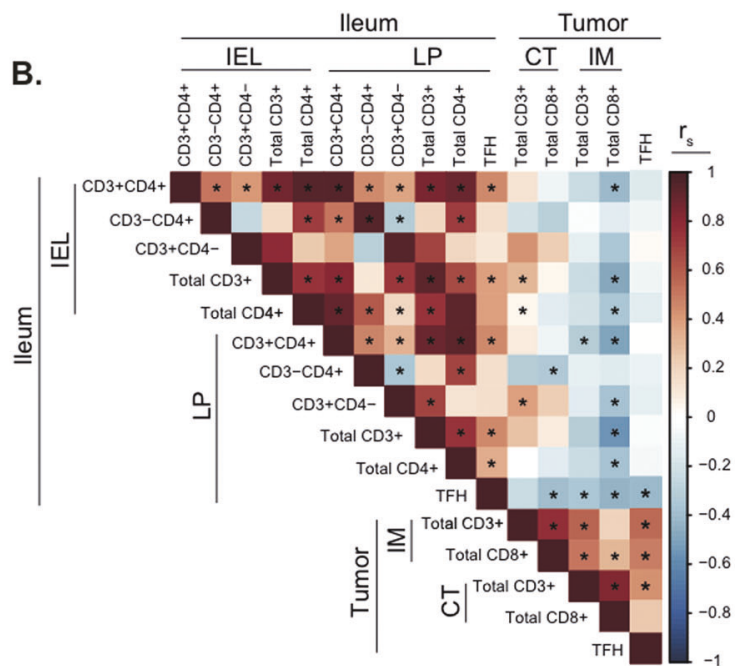

D.

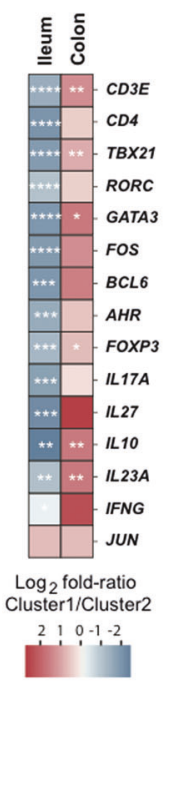

E. All tumor stages

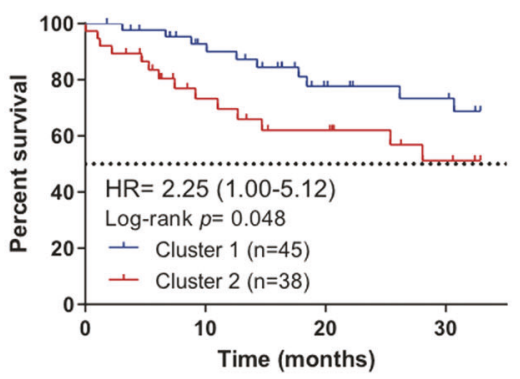

F. Stage IV patients

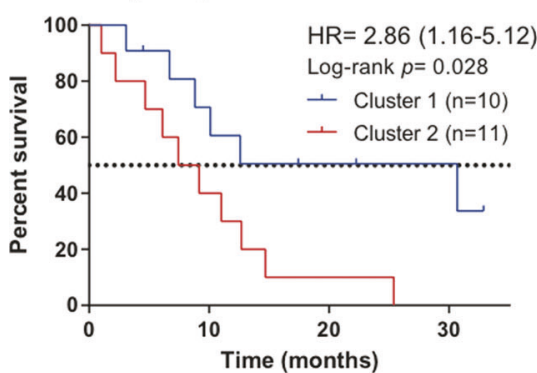

Fig. 1 Ileal immune parameters dictate pCC patients' prognosis. A Representative micrographs of (top panel) $\mathrm{CD}^{+} \mathrm{T}$-cell infiltrates in the tumor core by immunohistochemistry (IHC) with delineation of tumor areas by dotted black lines and (bottom panel) $\mathrm{CD}^{+}{ }^{+}$and $\mathrm{CD} 4^{+}$ T-cell infiltrates by immunofluorescence (IF) staining in autologous ilea of two distinct pCC patients. Scale bar $=100 \mu \mathrm{m}$. B Heatmap showing Spearman correlation coefficients between autologous tumor and ileum immune cell densities in 53 distinct pCC patients for whom paired samples were available. $* P<0.05$. IEL intra-epithelial lymphocytes, LP lamina propria, CT core of the tumor, IM tumor invasive margin. C Dendrogram and heatmap depicting the agglomerative hierarchical clustering of $\mathrm{pCC}$ patients ( $n=83$, columns) according to

Intrigued by these findings, we turned to mouse models to validate the observation that tumor progression could indirectly stimulate ileal inflammation/immunity. IEC-specific transgenic expression of the epithelial-mesenchymal transition regulator $\mathrm{Zeb} 2$ in mice $\left(\mathrm{Zeb} 2^{\mathrm{IEC}-\mathrm{Tg} /+}\right.$ mice) leads to ileal and colonic immune gene transcription (rows) at surgery. Distance was measured with 1-Pearson correlation coefficient and agglomeration with Ward's method. Clinical variables and tissue of origin are indicted by the corresponding color code on the top and side border, respectively. D Heatmap of gene expression patterns in autologous ileum and colon as a $\log _{2}$ fold ratio (FR) between cluster 1 and cluster 2 individuals. Mann-Whitney $U$ test: $* P<0.05$; $* * P<0.01$; $* * * P<0.001 ; * * * * P<0.0001$. E, F Kaplan-Meier curves for time to treatment failure (progression or cancer-related death) segregated according to the clustering from (C) analyzed by Mantel-Cox regression test in 83 pCC patients $(\mathbf{E})$ or only in stage IV metastatic pCC patients $(\mathbf{F})$.

increased intestinal permeability and spontaneous invasive colon carcinoma development in a microbiota-dependent manner [10]. At a young age, Zeb2 $2^{I E C-T g /+}$ mice develop dysplasia of the colon EP that progresses to neoplastic lesions [10], but maintain a normal small intestinal EP. Histological 
A.

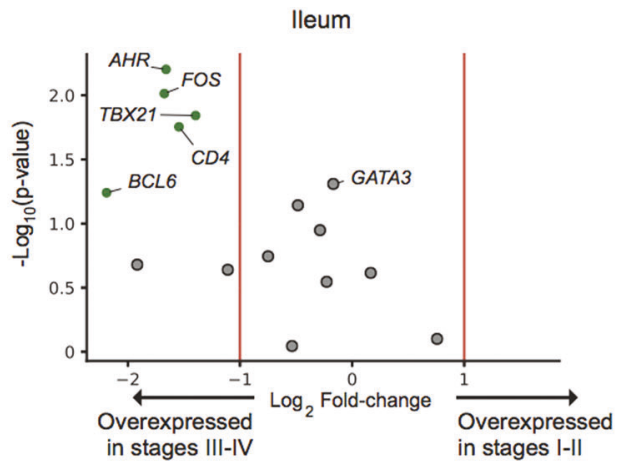

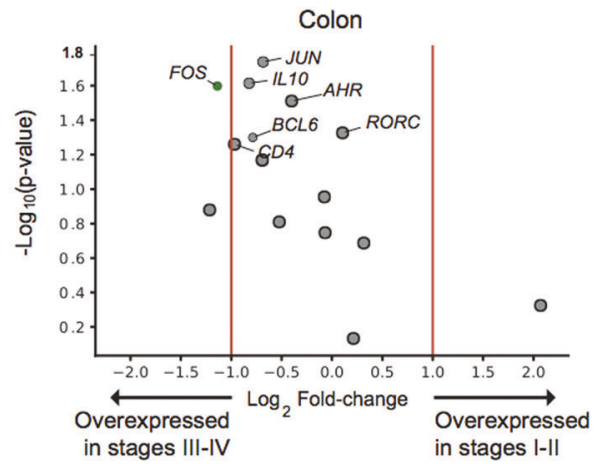

B.

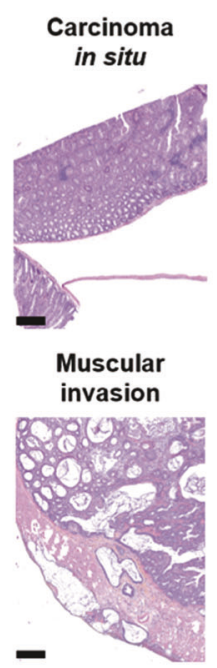

Scale bar $=200 \mu \mathrm{m}$

D.
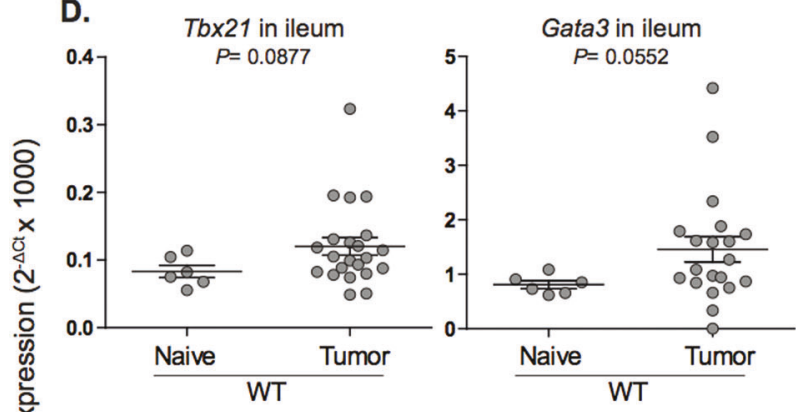

Sub mucosal

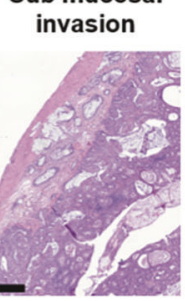

Peritoneal invasion

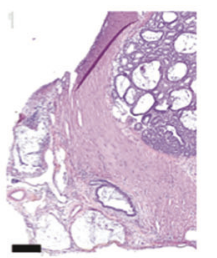

c.

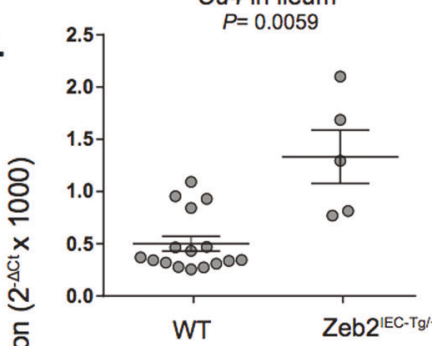

Foxp3 in ileum
$P=0.0012$

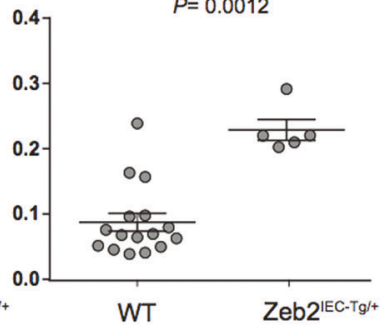

Rorc in ileum

$P<0.0001$
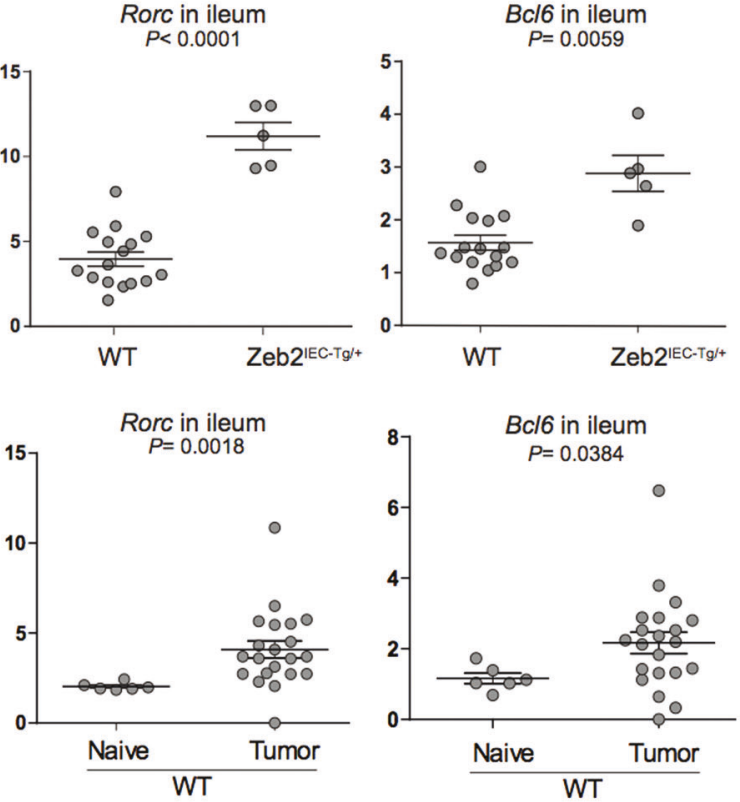
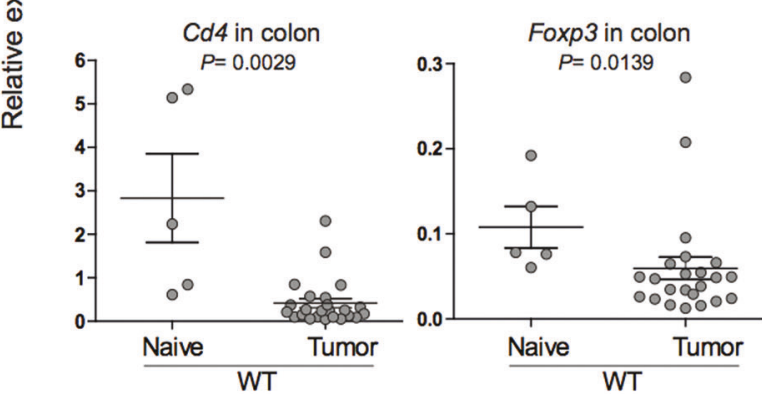

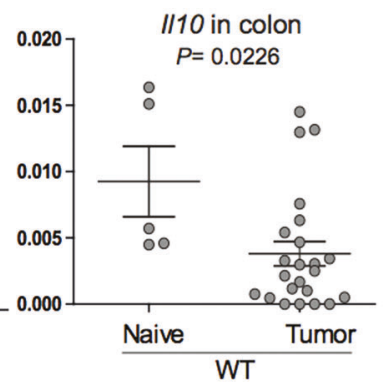

examination of colon tissues revealed different grades of tumor progression in individual mice. Among mucinous tumors in the proximal region of the colon, we observed cancers with clear signs of transmural invasion and growth throughout the muscularis mucosae until peritoneum invasion (Fig. 2B). The presence of invasive tumors in 
Fig. 2 Inverse relationship between ileal and cancer immune tonus in CC models. A Volcano plots of the differential immune gene transcription in qPCR of ileum (A) and colon (B) mucosae contrasting stage I-II versus stage III-IV pCC patients (TNM staging system). Volcano plots were generated computing for each gene product expressed in intestinal mucosae of 83 pCC patients: (i) the $\log _{2}$ of fold change (FC) among the mean relative abundances of transcripts after normalization in early versus advanced disease stages ( $x$ axis); (ii) the co- $\log _{10}$ of $P$ values deriving from Mann-Whitney $U$ test calculated on relative abundances in absolute values ( $y$ axis). Green and gray dots are considered significant $(P<0.062)$ or not $(P>0.062)$, respectively. B Representative micrographs of hematoxylin and eosin (H\&E)stained sections of intestinal samples collected from Zeb2 $2^{I E C-T g /+}$ mice showing different tumor progression. Scale bar: $50 \mu \mathrm{m}$. C Relative expression of immune genes in ilea of $\mathrm{Zeb2} 2^{I E C-T g /+}$ mice and WT littermates as assessed by RT-qPCR. One dot represents one ileum and the pooled data of all ilea are shown. Mean \pm SEM are depicted. Mann-Whitney $U$ test $P$ values are shown. D Relative expression of immune genes in ilea and colon of MC38-bearing mice WT versus naive WT mice as assessed by RT-qPCR. One dot represents one ileum or one colon and the pooled data of all ilea or colons are shown. Mean \pm SEM are depicted. Mann-Whitney $U$ test $P$ values are shown.

Zeb2 $2^{I E C-T g /+}$ mice was associated with increased expression of ileal immune genes (such as $C d 3 e, C d 4$, Ahr, Rorc, Bcl6, Gata3, IllO, Il23a, and Il27) compared to Zeb2 $2^{I E C-+/+}$ wildtype (WT) littermates (Fig. 2C, Supplementary Fig. S3A and Table S3). RT-qPCR examination of these tumor areas revealed a positive correlation between tumor burden and several immune genes found in ilea from pCC patients, such as $C d 4, B c l 6$, and Gata3 (Supplementary Fig. S3B).

We next investigated whether shifts in the gut microbiota might explain these ileal alterations during the colon carcinogenesis program. Of note, rederivation of Zeb2 $2^{I E C-T g /+}$ mice in germ-free (GF) conditions completely abrogated cancer development [10]. Mice reared in specific pathogenfree (SPF) conditions exhibited a more pronounced Th1/ Treg profile and an attenuated Il-17 tonus in their ilea as compared to axenic Zeb2 $2^{I E C-T g /+}$ (Supplementary Fig. S3C). However, during colon carcinogenesis, ilea from SPF Zeb2 $2^{I E C-T g /+}$ still manifested a relative overexpression in Illo, Gata3, and Il27 genes (Supplementary Fig. S3C and Table S4), suggesting that this ileal immunomodulation did not depend on the ileal microbiome.

To validate the notion that cancer can induce ileal inflammation, we switched to a transplantable colon tumor model that can be inoculated subcutaneously (s.c.) into syngeneic immunocompetent mice. We compared the ileal immune transcriptome of mice bearing s.c. MC38 cancers with that of naive tumor-free mice. Here again, we confirmed that the ileal mRNAs encoding Gata3, Bcl6, Rorc, and $T b \times 21$ were increased in tumor bearers at day 10 of implantation compared to naive counterparts. In contrast, Cd4, Foxp3, and 1110 transcription were reduced in the colon (Fig. 2D).
These results indicate that the presence of colon carcinogenesis is accompanied by a higher ileal immune tonus, paralleling tumor progression in human and mice.

\section{Chemotherapy alters the cancer-induced ileal immune activation}

The GALT provides specific types of immune responses to local challenges along the gastrointestinal tract. We recently showed that the ileal crypt cells were the main targets of OXA-based chemotherapy with respect to apoptosis induction [5]. Driven by this observation, we evaluated the impact of systemic cytotoxicants on the ileal immune signature.

Given that FOLFOX is the first-line standard chemotherapy for most patients with advanced CRC [2], we adapted this treatment to the mouse model, treating $Z e b 2^{I E C-T g /+}$ mice with a mixture of 5-FU and OXA. Histological examination of the colons and quantification of tumor invasion (as in Fig. 2B) revealed a mild response to therapy compared to PBS-treated controls (Fig. 3A), as expected given the aggressiveness of this model [10]. However, we did observe a chemotherapyinduced immune response in the TME with a significant increase in $\mathrm{CD}^{+}$cells, though no alteration in the frequency of $\mathrm{CD}^{+} \mathrm{T}$ cells (Fig. 3B, C). We found a negative correlation between the expression of ileal immune genes and the frequency of $\mathrm{CD}^{+}$TILs only in chemotherapy-treated mice (Fig. 3D), suggesting that chemotherapy partially dampened the ileal immune signature $(C d 4, T b x 21, I l 10$, and Il27) associated with carcinogenesis (Fig. 3E).

In conclusion, tumor infiltration showed a negative correlation with ileal immune tonus after chemotherapy in an aggressive spontaneous mouse model of colonic carcinogenesis.

\section{Bacteria regulate the antitumor efficacy of OXA by shaping the ileal immune profile}

Ileal apoptosis may affect the composition of the ileal microbiota and therefore influence immune responses in the GALT and tumor beds [5]. Therefore, we evaluated the fecal and ileal microbiota of our cohort of pCC patients [5]. An abnormal (or "dysbiotic") fecal microbial repertoire has been detected during adenoma-carcinoma transformation [10] as well as in CRC patients [11-13]. We performed $16 \mathrm{~S}$ rRNA gene sequencing of gene amplicons of ileal mucus from our previously described [5] pCC cohort $(n=70)$. Volcano plots revealed an overabundance of Prevotellaceae family members in ileal samples from patients diagnosed at advanced pCC stages (i.e., Prevotella buccalis and Prevotella denticola) compared with earlier stages (Supplementary Fig. S4). We next examined the ileal immune signature in relation to the ileal microbiome assessed by 
A.

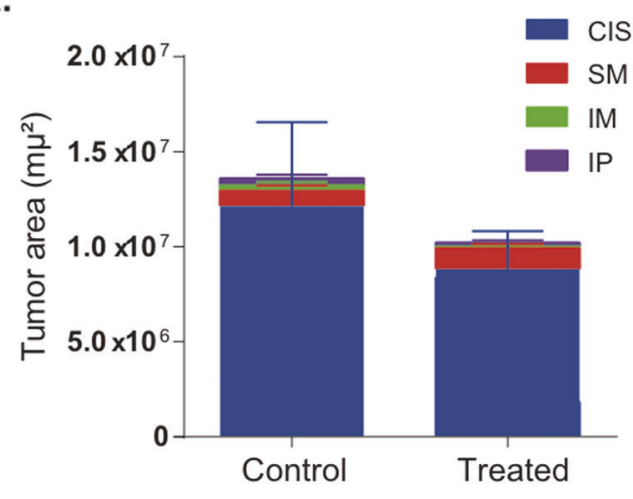

C.
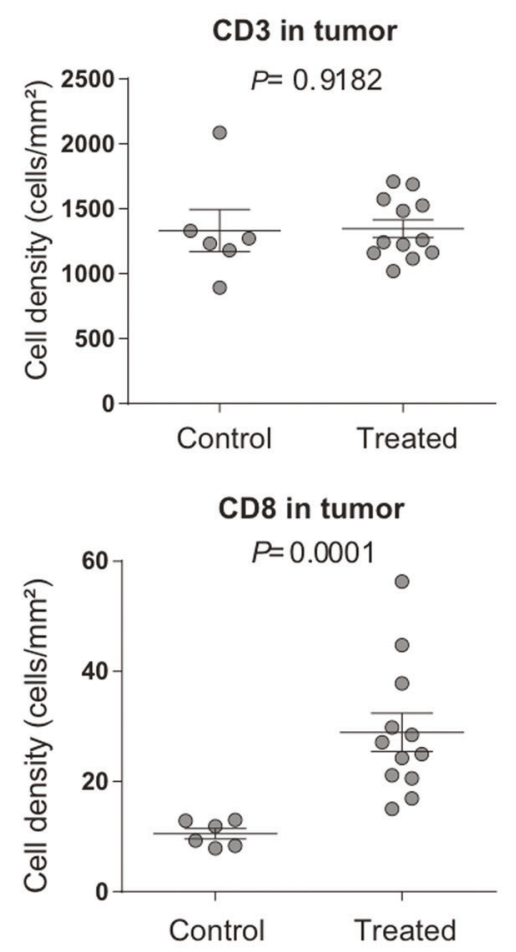

E.
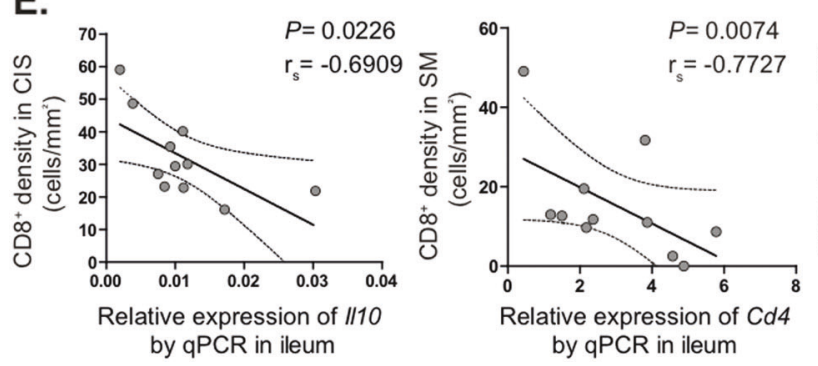

16S rRNA gene sequencing of the same patients. Surprisingly, the ileal microbiota could segregate patients classified according to their ileal immune gene expression. At the level of bacterial families or classes, Volcano plots
B.
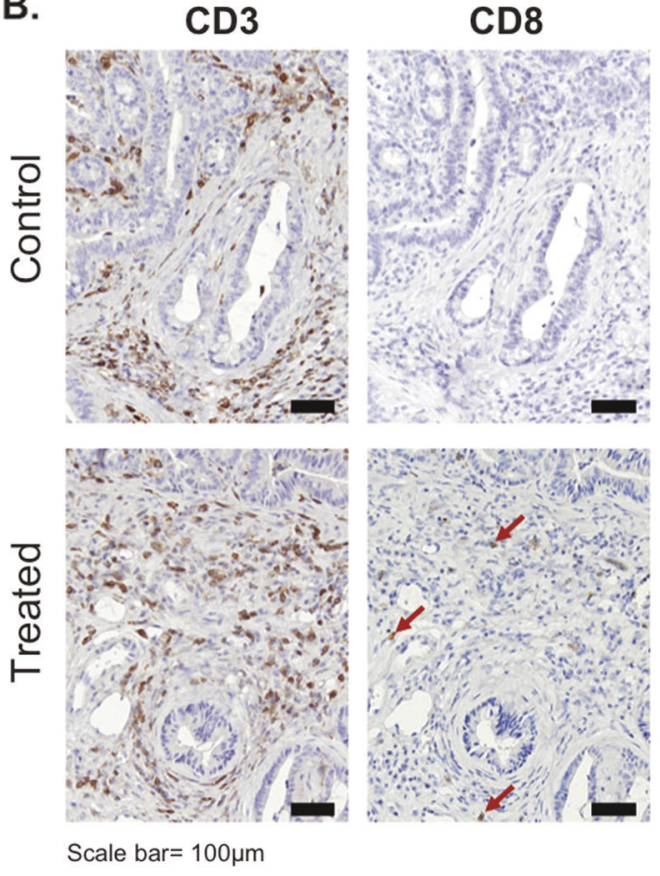

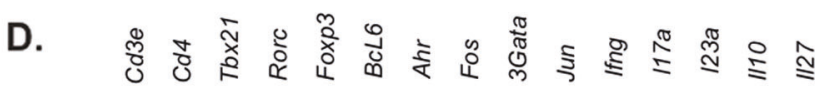

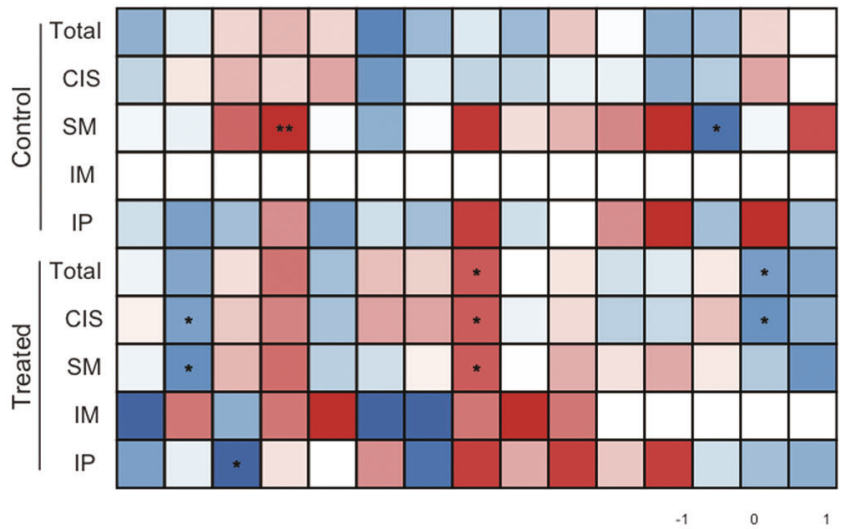

$r_{s}$
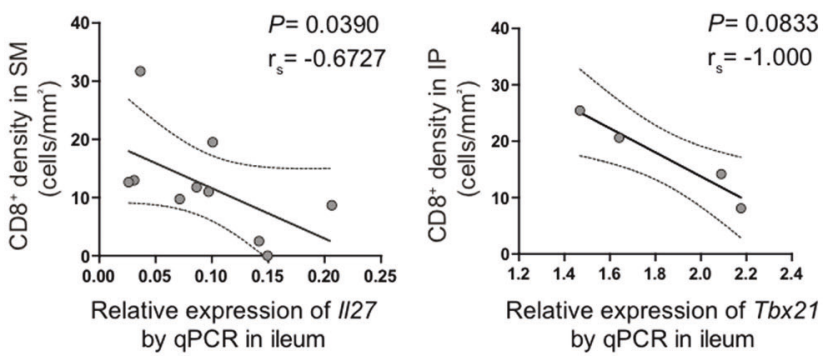

highlighted an overabundance of the Erysipelotrichaceae family and the Negativicutes class (such as Acidaminococcaceae, Selenomonadales unclass.) in cluster 1 patients (with better prognosis than cluster 2 patients) 
Fig. 3 Ileal immune parameters are restored by OXA-based chemotherapy. A Assessment of tumor loads by quantification of tumor areas in in situ, submucosal, muscular, and peritoneal tumors in $\mathrm{Zeb2} 2^{\mathrm{IEC}-\mathrm{Tg} /+}$ mice treated with $5-\mathrm{FU}+\mathrm{OXA}$ or vehicle (PBS control). B Representative micrograph of $\mathrm{CD}^{+}$(left panel) and $\mathrm{CD}^{+}$(right panel, indicated with arrows) cell infiltrates by IHC staining in the tumors of Zeb2 $2^{I E C-T g /+}$ mice treated by 5-FU + OXA or PBS control. Scale bar: $50 \mu \mathrm{m}$. C Quantification of $\mathrm{CD}^{+}$(upper panel) and $\mathrm{CD} 8^{+}$ (lower panel) cell densities in tumors of $\mathrm{Zeb2} 2^{I E C-T g /+}$ mice (relative to IHC staining in B). Mann-Whitney $U$ test $P$ values are shown. D Heatmap showing Spearman correlation coefficients between ileum immune gene expression and $\mathrm{CD}^{+}$cell densities in tumors (quantified in C) of Zeb2 $2^{I E C-T g /+}$ treated by 5-FU + OXA (or PBS control). CD8 ${ }^{+}$ TILs were assessed by IHC and quantified in tumor areas with different degree of invasion. Significant correlations are indicated, $* P<$ 0.05. CIS carcinoma in situ, SM submucosal tumor, IM muscleinvasive tumor, IP invasive tumor in the peritoneum; Total: total tumor area. E Spearman correlation between ileum immune genes and CD8 ${ }^{+}$ cell densities in tumor. CD8 ${ }^{+}$TILs were assessed by IHC and quantified in tumor areas with different degrees of invasion. One dot represents one mouse; the black and dotted lines show the regression line and $95 \%$ confidence intervals, respectively. Spearman coefficients and $P$ values are shown in the figures. CIS carcinoma in situ, SM submucosal tumor, IP invasive tumor in the peritoneum.

(Fig. 4A, left panel). At the species level, there was a significant enrichment in Prevotella spp. (P. oralis, P. oryzae) in cluster 2 patients with dismal prognosis compared to cluster 1 (Fig. 4A, right panel). Conversely, the only bacterium enriched in the favorable cluster 1 (compared with cluster 2) was Bacteroides fragilis (Fig. 4A, right panel), in line with the previously reported association of this species with an immunogenic ileal microbiota [5].

To establish a cause-effect relationship between the microbiota composition and the ileal prognostic signature, we took advantage of the "avatar" model in which GF mice (previously shown to be nonresponders to OXA [14]) received fecal microbiota transplantation (FMT) from human pCC patients and hence were "humanized" with respect to a potentially pathogenic intestinal microbiota [5]. In these gut experiments of "humanization", some patient feces (8/12 cases) yielded an avatar responder (aR) phenotype in the mice, meaning that subsequently implanted MC38 responded to OXA treatment, while others yielded an avatar nonresponder phenotype (aNR) [5]. OXA-treated aR (but not aNR) mice showed a depletion of the mRNAs coding for Cd4, Tbx21, Rorc, Ahr, Bcl6, and Foxp3 from their ilea (Fig. 4B). This difference between aR versus aNR transcriptomes was only found in the ileum, not in the colon (Fig. 4B and Supplementary Table S5).

Of note, we previously reported that aR mice exhibited a stronger increase in Tfh cells in tumor- draining lymph nodes (tdLNs) than aNR mice [5]. The abundancy of Tfh in tdLN modulated by FMT negatively correlated with the expression of Bcl6 in the ileal mucosa (Fig. 4C, left panel), confirming the negative relationship between ileal and tumor-specific immunity outlined in Fig. 1 in patients.
Conversely, tdLNs from aNR mice contained higher $\mathrm{CCR}^{+}{ }^{+} \mathrm{CXCR}^{-}{ }^{-} \mathrm{CD}^{+}{ }^{+}$Th17 cells (known to be associated with an immuno-suppressive milieu [15]) than those from aR mice, and such cells exhibited a positive correlation with ileal $A h r$ mRNA levels, AhR being a transcription factor associated with Th17 differentiation [16] (Fig. 4C, right panel).

Ileal immune responses mainly take place in mLNs where $\mathrm{T}$ cells acquire a gut tropism and relocalize within intestinal EP or LP [17]. However, lymphocytes can dynamically migrate through the lymphatic space to find a cognate antigen in other locations, including extraintestinal tumor sites by various homing mechanisms [18]. To test the impact of the microbiota in altering migration patterns of lymphocytes primed in the gut, we performed oral gavages with bacteria associated with favorable (such as Bacteroides fragilis) or dismal prognosis (such as Paraprevotella clara). We administered this oral supplementation twice, 24 hours before and after OXA i.p. injection. Here again, we found a mirror image of the leukocyte infiltration induced by orally administered bacteria in the ileal mucosa and the tumor bed. B. fragilis was associated with higher TILs and lower $\mathrm{CD}^{+} 5^{+}$cells in the ileal compartment, compared to P. clara-treated groups (Fig. 4D).

The aforementioned results support the notion that the ileal microbiota shapes local immune responses, which then control colon cancer immunosurveillance.

\section{Discussion}

Here, we demonstrate that the prognosis of pCC patients is associated with an ileal immune fingerprint that inversely correlates with the colonic signature and appears to be linked to tumor immunosurveillance.

First, we highlighted in a cohort of $83 \mathrm{pCC}$ patients that those individuals presenting with a low ileal immune gene transcriptome have a favorable prognosis, even when diagnosed with a stage IV pCC (Fig. 1C, F). Second, the $\mathrm{CD} 3{ }^{+} \mathrm{CD} 4^{+} \mathrm{T}$ cells of IELs and LP inversely correlated with $\mathrm{CD}^{+} \mathrm{CD}^{+}$counts, a central parameter of the immunoscore [7] (Fig. 1B). Indeed, scarce expression of immune genes in the ileum corresponded to high immune gene expression in healthy colons, specifically for gene products of the Th1 (CD4, TBX21), Th2 (GATA3), and Treg (FOXP3, IL10) differentiation profile, while the Th17 pattern (centered around ILI7A, AHR, and RORC) was relatively stable across the small and large parts of the intestine (Supplementary Fig. 2). This might account for the colon cancer infiltration of those Th1/Th2 cells in the core of the tumor in favorable cases.

Conversely, the ileal immune tonus was affected by colonic carcinogenesis as indicated by the fact that the since 
A.

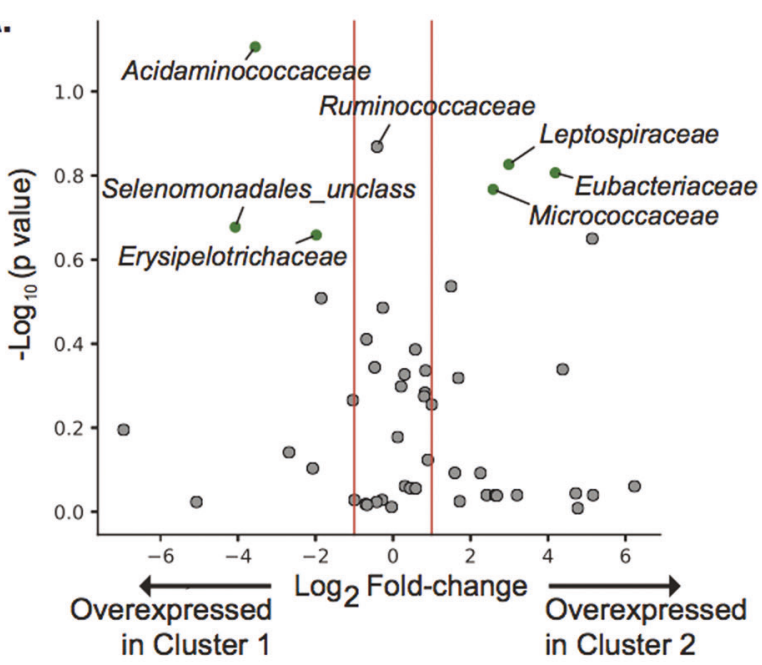

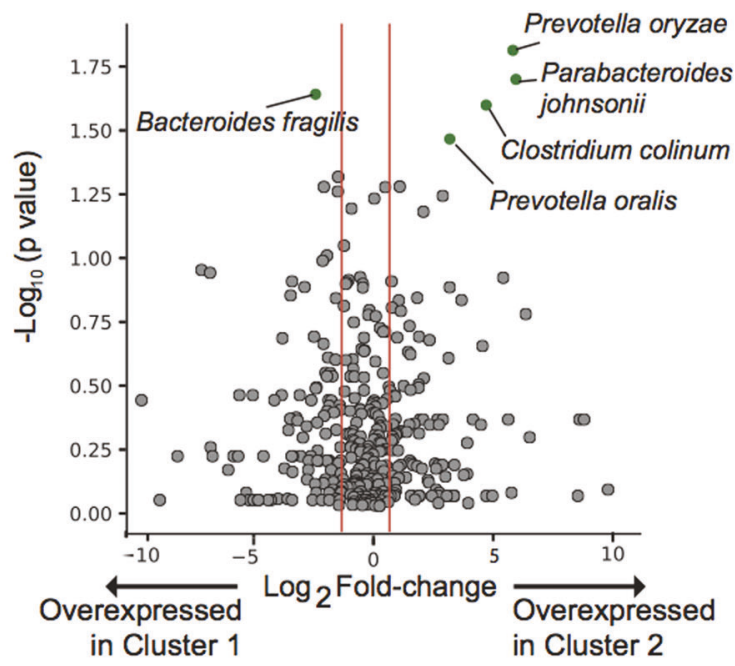

B.

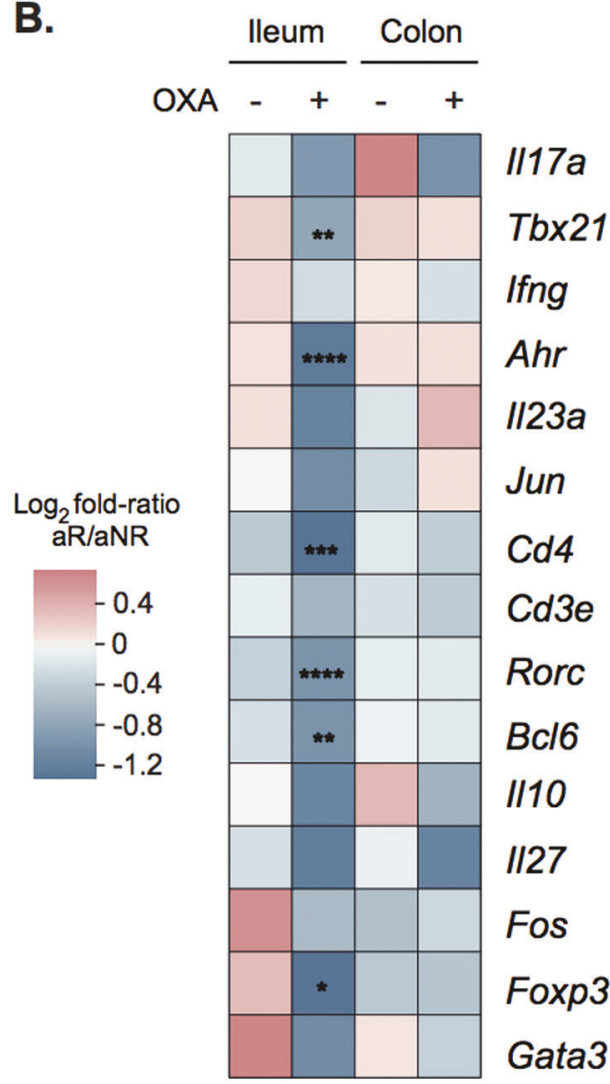

growth of heterotopic (s.c. MC38) or orthotopic (EMTinduced Zeb2-transgenic) colon cancers induced this upregulation of ileal immune gene products (Fig. 2C, D). At this point, it remains an open question how tumors can send proinflammatory signals to the ileum.

We also found evidence that OXA-based chemotherapy reshuffles the ileal immune system, favoring the reduction of immune transcription of a variety of gene products in MC38-bearing avatar mice as well as in Zeb2-transgenic animals (Figs. 3 and 4). The striking negative correlations
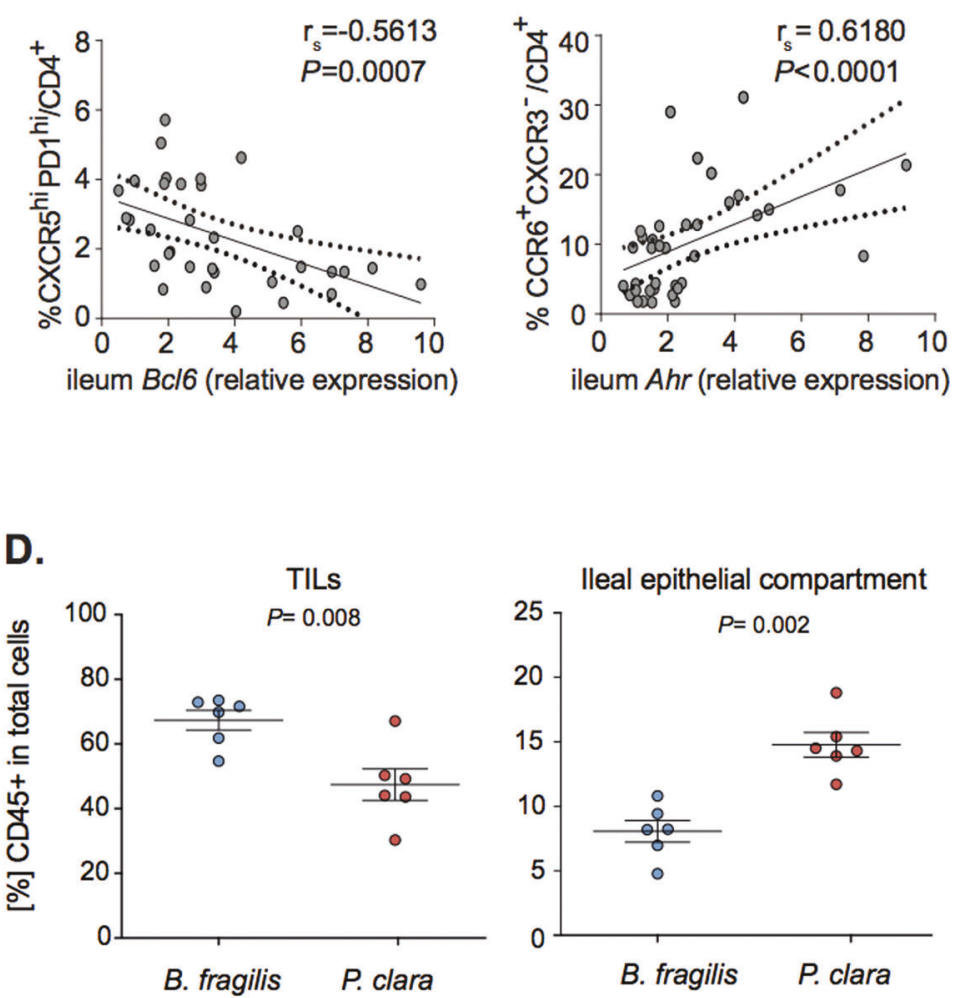

found in the Zeb2 $2^{I E C-T g / 4}$ and s.c. MC38-transplantable models between the ileal and tumor immunity post-OXAbased therapy suggest that antigen-specific $\mathrm{T}$ cells could be primed in mLNs and migrate back to tumor beds instead of returning to the inflamed ileal mucosa. Although speculative, this is in line with our previous findings obtained in s.c. MC38 in which OXA generated an immunogenic cell death of ileal IEC that contributed to elicit Tfh in mLNs and tdLNs and fosters $\mathrm{CD} 8^{+}$T-cell-dependent tumor control [5]. We reported that OXA treatment triggered the 
Fig. 4 Microbiome composition regulates the antitumor efficacy of OXA by shaping ileal immune profile in experimental models. A Volcano plot representation of differential microbiota composition at family (A) and species (B) levels matching cluster 1 versus cluster 2 pCC patients as defined in Fig. 1C. Volcano plots were generated computing for each bacterial family (left) or species (right) residing in ileal mucosae of 83 pCC patients: (i) the $\log _{2}$ of FR among the mean relative abundances in cluster 1 versus cluster 2 ( $x$ axis); (ii) the co$\log _{10}$ of $P$ values deriving from Mann-Whitney U test calculated on relative abundances ( $y$ axis). Green dots are considered significant at $P<0.05$ (gray dots $p>0.05$ ). B. Heatmap of the $\log 2$ FR of the immune gene transcript levels between aNR and aR wild-type mice in PBS and OXA-treated groups in ilea and colons at sacrifice (day 21). Mann-Whitney $U$ test: $* P<0.05$; $* * P<0.01$; *** $P<0.001$; **** $P<$ 0.0001. C Spearman correlations between ileal Bcl6 gene transcripts and percentages of Tfh (left panel) and between ileal Ahr gene transcripts and percentages of $\mathrm{CCR} 6^{+} \mathrm{CXCR}^{-} / \mathrm{CD}^{+}{ }^{+} \mathrm{T}$ cells determined by FACS in tdLN in OXA-treated avatar groups at sacrifice (day 21 after treatment). The black and dotted lines show the regression line and $95 \%$ of confidence intervals, respectively. Spearman coefficients and $P$ values are shown in the figures. D Flow cytometry analysis of $\mathrm{CD}_{4} 5^{+}$lymphoid cell infiltration in tumor and ileal epithelial compartment of MC38 tumor-bearing mice treated with OXA and gavaged with $B$. fragilis or $P$. clara. Each dot represents one tumor or one ileum at sacrifice (day 4 after treatment). Means \pm SEM are depicted. Mann-Whitney $U$ test $P$ values are shown.

migration of ileal CD11 $\mathrm{b}^{-} \mathrm{CD} 103^{+}$LP-DCs to the $\mathrm{mLN}$ and their upregulation of IL-12 and IL-1 $\beta$ in a gut microbiotadependent manner [5]. We also reported that migratory LPDCs from the terminal ileum contributed to Tfh priming post OXA [5]. It is tempting to speculate that $\mathrm{T}$ cells primed in $\mathrm{mLN}$ may either traffic back to the ileal inflammation caused by OXA-induced apoptosis of the crypts or instead are redirected to tumor lesions. It is conceivable that the chemokine axis is reshuffled by cytotoxic agents in both ileal and tumor epithelia in an inversed manner, changing the homing equilibrium between gut to tumor beds, as previously shown for CCR9 ${ }^{+} \mathrm{T}$ cells $[19,20]$.

Functional links between ER stress in the epithelial barrier and immune activation in the TME were recently reported in $R n f 5^{-/}$mice [21]. RNF5 is a membraneanchored E3 ubiquitin ligase implicated in endoplasmic reticulum (ER)-associated protein degradation. Decreased secretion of antimicrobial peptides and increased cell death in the ileal crypts caused intestinal dysbiosis in $R n f 5^{-/}$ mice. This bowel injury allowed the recruitment, activation, and mobilization of CCR7-expressing dendritic cells to Peyer patches, mLN [22], and tdLN, culminating in the massive infiltration of IFN $\gamma$-producing $\mathrm{CD}^{+}$and $\mathrm{CD} 8^{+}$ TILs and tumor control of poorly immunogenic melanoma [21]. The microbiota shift in Rnf5 ${ }^{-/-}$mice was underscored as a key factor in the immunosurveillance mechanisms. This report is in line with our findings showing that some intestinal commensals can affect the ileal immune system during OXA-induced cancer immunosurveillance. By segregating responders from nonresponders in "humanized" mice bearing colon cancers, we demonstrated that the OXAinduced reduction of tumor size was associated with low ileal immune transcription (Fig. 4B, C), paralleling Tfh accumulation and Th17 reduction in tdLNs in responders. Patients diagnosed with $\mathrm{pCC}$ belonging to cluster 1 (displaying a low ileal immune profile, Fig. 1C, D) presented a relative dominance of $B$. fragilis and an underrepresentation of Prevotella spp., these two species creating an opposite balance of the leukocyte infiltrates in the ileum and tumors (Fig. 4D). In our previous work [5], we showed that $B$. fragilis contributes to IL-12- and IL-1 $\beta$-dependent priming of Tfh in tdLNs. Other reports proposed alternative mechanisms on how microbial species could modulate T-cell patrolling patterns during carcinogenesis. Microbial species may induce a burst of chemokines in GALT or in the TME, thus affecting the trafficking of helper and effector T cells [21]. In CRC, the commensals may act on cancer cells to modulate their capacity to produce chemokines that may govern the immunoscore and patient prognosis through the recruitment of Th1, Tfh, or Th17 cells into the tumor bed [23]. In hepatocellular carcinoma, the gut microbiome uses bile acids as messengers to regulate chemokine CXCL16 levels on liver sinusoidal endothelial cells in order to control the accumulation of $\mathrm{CXCR}^{+}$hepatic natural killer T (NKT) cells that keep liver tumors at bay [24]. In contrast, lipoteichoic acid, a cell wall component of Gram-positive bacteria, enhances the senescence-associated secretory phenotype of hepatic stellate cells in collaboration with a gut microbial metabolite, deoxycholic acid, and upregulates the inflammatory cascade and $\operatorname{COX} 2$ in a TLR2-dependent manner. In turn, COX2-mediated prostaglandin E2 production suppresses the antitumor immunity, thereby contributing to hepatocellular carcinoma progression [25].

Previous reports using oral administration of anticancer probiotics analyzed the immunomodulatory effects of these live biotherapeutics on the colonic mucosae, highlighting the accumulation of IFN $\gamma$-producing $\mathrm{CD}^{+} \mathrm{T}$ cells as a surrogate marker of tumor immunosurveillance of cancers established in gut or extraintestinal locations. Tanoue et al. used a 11-strain-based product that elicited potent cytotoxic $\mathrm{T}$-cell responses, including tissue-resident $\mathrm{T}$ cells devoid of colitis-inducing properties that were associated with ICIinduced anticancer effects against melanoma [26]. Wang et al. administered Akkermansia muciniphila and its bacterial TLR4 derivative A-muc_1100 that both prevented colitis-induced CRC and CT26 by inducing the colonic accumulation of $\mathrm{TNF} \alpha$-producing $\mathrm{CD} 8^{+} \mathrm{T}$ cells elicited in $\mathrm{mLN}$ and spleens [27]. In these reports, including ours, it is unclear to which extent these CTL responses elicited from the gut and redirected to TME contribute to the antitumor effects. Some of these CTL responses might be specific for bacterial antigens, sharing some molecular mimicry with 


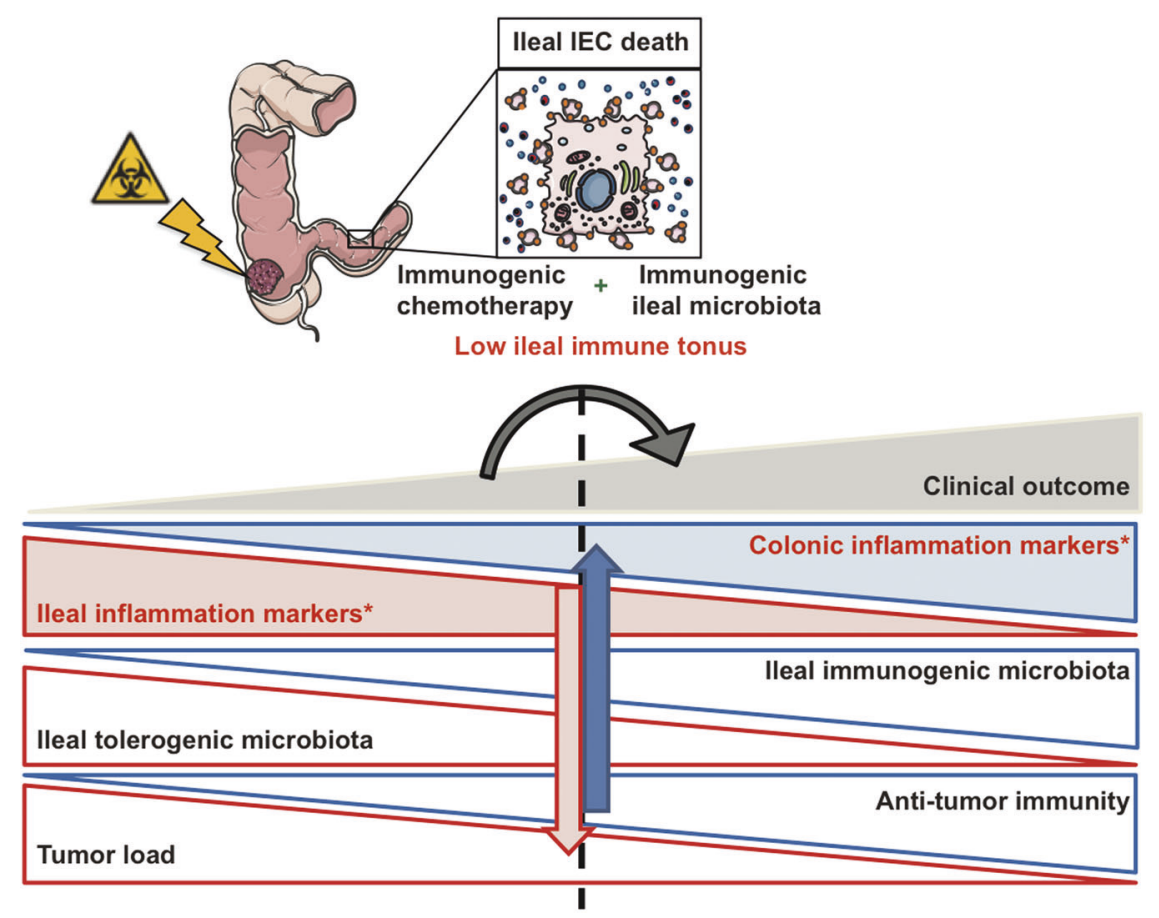

Fig. 5 The interactive dialog between the microbiota, the immune system, and the epithelial barrier in the ileal mucosae of colon cancer patients. Ileal IEC apoptosis together with natural adjuvants from distinct commensals elicit an immune response against selfantigens (likely derived from crypt/villus-progenitor cells) that eventually infiltrates proximal colon cancers (pCC) as demonstrated in Roberti et al. [5]. Here we show the relevance of ileal mucosal immune transcriptome on the prognosis of pCC patients and its connection with ileal microbiota. In advanced staging or nonresponders to adjuvant therapies, the whole immune ileal transcriptome remains upregulated, while immune gene products encoding transcription factors and cytokines are downregulated in the healthy colon mucosae. This mirror image between ileum and colon of the inflammation and immunity fingerprints in the lamina propria and/or epithelial areas may reflect a coordination of the two compartments to face the challenge of tumor progression. The empty boxes refer to our previous work reported in Roberti et al. [5]. The colored boxes and arrows with stars* represent the follow-up study proposed in this paper.

fingerprint (immune gene expression pattern and microbiome composition) to address the prognosis of metastatic patients. This signature can be routinely studied upon pCC surgery, taking advantage of the systematic resection of the terminal ileum. Further prospective work is ongoing in our laboratories to validate these novel biomarkers in MSI and MSS CRC. In addition, our present and previous [5] findings strongly advocate for the use of ileum and crypttargeting cell death inducers in combination with such immunotherapies to convert "cold" into "hot" pCCs, regardless of their MSI status.

\section{Materials and methods}

\section{Patients}

In a cohort of pCC patients who underwent hemicolectomy, we previously reported the epithelial and microbial parameters that were significantly associated with outcome [5]. The inclusion criteria for the study were patients with colon adenocarcinoma who underwent right hemicolectomy plus 
ileal resection (last 10 centimeters). The number of samples was selected based on availability of the samples. Patients were not on antibiotics at the moment of surgery. Four different health cancer centers participated in the study: Gustave Roussy Cancer Campus (Villejuif, France), Centre G-F Leclerc (Dijon, France), Hôpital Bicêtre (Le KremlinBicêtre, France), and Centre hospitalier universitaireEstaing (Clermont Ferrand, France). Clinical studies were conducted according to the ethical guidelines and approval of the local CCPPRB. The study received the legal authorization by the Ministère de l'Enseignement Supérieur et de la Recherche (AC-2013-1884). Written informed consent in accordance with the Declaration of Helsinki was obtained from all patients.

\section{Patients' clinical categorization and follow-up}

The information pertaining to tumor staging (TNM staging system of the American Joint Committee on CancerAJCC) and MSI status was obtained in pathology reports. Information pertaining to cancer recurrence, death, and causes of death was obtained from hospital records. All observations were censored at loss to follow-up and at the end of the study period. OS was measured from the date of surgery to the date of death from any cause. Time to treatment failure (TTF) was measured from the date of surgery to the date of local or regional recurrence, distant metastases, or death from cancer and treatment-related death; patients were censored at non-cancer-related death (TTF study period 1000 days).

\section{Preparation of patients' samples for the study}

Samples were collected at the time of surgery. Fecal content from colon for FMT experiments and mucus adherent to the ileum mucosae for $16 \mathrm{~S}$ rRNA gene sequencing were already described [5]. Mucosal biopsies: approximately 4 $\mathrm{cm}^{2}$ of ileal and nontumoral colonic tissue (distant from the tumor) that included at least the submucosa was removed, cut into small pieces (approximately $1 \mathrm{~cm}^{2}$ ), and transferred in sterile cryotubes. All samples were immediately frozen at $-80^{\circ} \mathrm{C}$ after collection. Tumor samples were retrieved from formalin-fixed paraffin-embedded (FFPE) tissue from the pathology archives. Paired analyses were done with the maximal number of available samples; there were no exclusions, except for samples that did not meet the quality control criteria. Patients' clinical characteristics are detailed in Table S1.

\section{Mice}

Female C57BL/6 mice were purchased from Harlan. Mice were used between 7 and 14 weeks of age. GF C57BL/6 were obtained from the facility located at CDTA (Cryopreservation, Distribution, Typage et Archivage, Orléans, France). Zeb2 $2^{I E C-T g /+}$ mice were previously described [10].

Axenic mice were housed in positive-pressure isolators. All experiments were performed on mice of C57BL/6 genetic background. Mice were housed in individually ventilated cages in a SPF animal facility. Experiments were performed at the animal facility in Gustave Roussy Cancer Campus, at the VIB Center for Inflammation Research and the GF facility of Ghent University. All experiments on mice were performed in compliance with institutional and European laws and regulations (Ministère de la Recherche, de l'Enseignement Supérieur et de l'Innovation, MESRI, and Ethical Committee for Animal Experimentation at Ghent University's Faculty of Sciences and Faculty of Medicine and Health Sciences). Sample sizes were decided based on previous publications and experience and common standards in similar field, for calculating statistical significance. No animals were excluded from the analysis.

\section{Tumor immune profiling by immunohistochemistry in human samples}

These results were previously reported [5]. In the present study, we reanalyzed them $(n=53)$ to perform correlations with the ileum immunity.

\section{Immunofluorescence staining, scanning, and analysis for CD3 and CD4 expression in ileum}

For multiplexed staining, $3-\mu \mathrm{m}$-thick sections of formalinfixed, paraffin-embedded ileal tissue were stained by automated immunostainer (DISCOVERY ULTRA, Ventana, IGR). Heat-induced antigen retrieval in Citrate buffer $(\mathrm{pH}$ 6.0) for 10 minutes at $100^{\circ} \mathrm{C}$ was performed. Then, the slides were incubated on primary monoclonal rabbit antihuman CD4 antibody (Spring, SP35, $0.5 \mu \mathrm{g} / \mathrm{mL}$ ) for 1 hour at $37^{\circ} \mathrm{C}$, detected by Discovery UltraMap anti-rabbit HRP (Ventana, \#760-4315), and visualized by Discovery Cy5 kit (Ventana, \#760-238). Heating step with Citrate Buffer was carried out, as described above. Next, the slides were incubated on primary polyclonal Rabbit anti-human CD3 antibody (DAKO, \#A0452, $3 \mu \mathrm{g} / \mathrm{mL}$ ) for $1 \mathrm{~h}$ at $37^{\circ} \mathrm{C}$, detected by Discovery UltraMap anti-rabbit HRP (Ventana, \# 760-4315), and visualized by Discovery FAM kit (Ventana, \# 760-243). After the heating step with Citrate Buffer, nuclei were subsequently visualized with Spectral DAPI (Perkin Elmer, FP1490, 1:10). Fluorescence analysis. Images displayed in the figures were acquired as whole-slide images (WSI) with a slide scanner Zeiss Axio Scan.Z1 (objective Plan-Apochromat 20×/0.8, 3CCD camera Hitachi HV-F202SCL) and exported from the Zeiss Zen 2 lite software as TIFF images. Some of the WSI were processed 
with using an algorithm developed in Visiopharm Integrator System (VIS) (Visiopharm A/S, Denmark). ROI was defined for each WSI by applying a threshold on the DAPI intensity, and then mean fluorescence intensity was measured in those ROIs.

\section{Characterization of gut immune gene expression profile by real-time quantitative PCR analysis}

Total RNA from gut biopsies was extracted with RNeasy Mini Kit (Qiagen) and then reverse-transcribed into cDNA with the SuperScript III Reverse Transcriptase and the RNaseOUT ${ }^{\mathrm{TM}}$ Recombinant Ribonuclease Inhibitor (Life Technologies, Saint Aubin, France), in the presence of random primers (Promega, Charbonnieres, France) and the Deoxynucleoside Triphosphate Set, PCR grade (Roche Diagnostics, Meylan, France). cDNA was analyzed by real-time quantitative PCR (RT-qPCR) with the TaqMan method with TaqMan Gene Expression Assays using the Universal Master Mix II (Invitrogen) according to the manufacturer's instructions using the 7500 Fast Real-Time PCR system (Applied Biosystems). Expression was normalized to the expression of the housekeeping gene of Beta 2 Microglobulin by means of the $2^{-\Delta \mathrm{Ct}}$ method. All primers were from TaqMan Gene Expression Assay (Thermo Fisher).

Mouse primers: $B 2 m$ (Mm00437762_m1), Cd3e (Mm01179194_m1), Cd4 (Mm00442754_m1), Tbx21 (Mm00450960_m1), Ifng (Mm01168134_m1), Rorc (Mm01261022_m1), Il17a (Mm00439618_m1), Foxp3 (Mm00475162_m1), Il10 (Mm01288386_m1), Gata3 (Mm00484683_m1), Il27 (Mm00461162_m1), Il23a (Mm00518984_m1), Bcl6 (Mm00477633_m1), Ahr (Mm00478932_m1), Fos (Mm00487425_m1), and Jun (Mm00495062_s1).

For studies comparing naive mice to MC38-bearing WT mice: MC38 bearers bearing WT mice $n=21$ and naive WT $n=6$ mice have been tested.

For studies comparing WT mice (littermate controls) to Zeb2 $2^{I E C-T g /+} n=16$ samples from WT mice and $n=$ 5 samples from $Z e b 2^{I E C-T g /+}$ have been tested.

For studies comparing GF mice to SPF mice, WT SPF mice $n=16$, WT GF mice $n=7, Z e b 2^{I E C-T g /+}$ SPF mice $n=$ 5 , and Zeb2 $2^{I E C-T g /+}$ GF mice $n=9$ have been tested.

For studies characterizing gut immune gene expression profile in different tumor locations, nontreated $\mathrm{Zeb} 2^{I E C-T g /+}$ mice $n=6$ and treated Zeb2 $2^{I E C-T g /+}$ mice $n=12$ have been tested.

Human primers: B2M Forward: 5'-GATGAGTATGCCT GCCGTGT-3'; B2M Reverse 5'-AATTCATCCAATCCAA ATGCG-3'; $B 2 M$ Probe 5'-(6FAM) AACCATGTGACTTT GTCACAGCCCAA(TAM)-3', CD3E (Hs01062241_m1), CD4 (Hs01058407_m1), TBX21 (Hs00894392_m1), IFNG (Hs00989291_m1), RORC (Hs01076112_m1), IL17A
(Hs00174383_m1), FOXP3 (Hs01085834_m1), IL10 (Hs00961622_m1), GATA3 (Hs00231122_m1), IL27 (Hs00377366_m1), IL23A (Hs00372324_m1), BCL6 (Hs00153368_m1), AHR (Hs00169233_m1), FOS (Hs04194186_s1), JUN (Hs01103582_s1). A total of $n=$ 83 patients have been tested.

Analyses were done with the maximal number of available samples; there were no exclusions, except for samples that did not meet the quality control criteria.

\section{Chemotherapeutic treatment of Zeb2-transgenic mice}

All chemotherapies were obtained from the Gustave Roussy Cancer Campus. Mice were allocated to groups ensuring an equal distribution of genders. When mice were 6 weeks old, 5-FU and OXA were administered i.p. at $50 \mathrm{mg} / \mathrm{kg}$ and $10 \mathrm{mg} / \mathrm{kg}$, respectively. A second treatment with OXA was performed at week 10 with the same dose $(n=12)$. Mice from control groups were injected with PBS $(n=6)$. Mice were euthanized at week 15 to examine drug effects. One experiment was performed for IHC analysis and two independent experiments were performed to analyze ileal gene expression (one comprising GF Zeb2-transgenic mice).

\section{Tumor immune profiling by immunohistochemistry in the Zeb2-transgenic model}

Immunohistochemistry staining, scanning, and analysis of double-staining CD8-CD3. Rabbit anti-mouse CD8 $\alpha$ monoclonal Ab (Cell signaling, \#D4W2Z, 1:400) and rabbit antimouse CD3 polyclonal Ab (Dako, \#IS503) were performed on 3- $\mu$ m-thick sections of formalin-fixed, paraffin-embedded colon from murine samples (nontreated mice, $n=6$, and treated mice, $n=12$ ). Antigen retrieval was performed by incubating slides in Tris-EDTA ( $\mathrm{pH} \mathrm{8.0)}$ for $30 \mathrm{~min}$ at $98^{\circ} \mathrm{C}$. Then the antibodies were successively incubated for $1 \mathrm{~h}$ for CD8 and CD3 at room temperature, and detected respectively by PowerVision poly HRP anti-rabbit IgG (MM France, \#PV6119). These slides were revealed by DiAminoBenzidine (DAKO, \#K3468). Finally, the sections are counterstained by Hematoxylin (RAL Diagnostics, \#3205501000). Images displayed in the figures were acquired as WSIs with a slide scanner Olympus VS120 at 20x objectives. For mouse tissue analysis, QuPath software was used [37]. Regions of interest (ROIs) of normal tissue or tumor were defined by two pathologists (SY and $\mathrm{PO}$ ). $\mathrm{CD}^{+}$or $\mathrm{CD}^{+}$cells were identified using QuPath software.

\section{FMT experiments}

Tumor growth and FACS analysis of tdLN were reported before [5]. 


\section{Subcutaneous model of MC38 for therapy settings}

MC38 cell line was derived from methylcholanthrene-induced murine colon adenocarcinoma cells (NCI, Bethesda, MD) and tested negative for mycoplasma contamination. Syngeneic C57BL/6J mice were implanted with $1 \times 10^{6} \mathrm{MC} 38$ WT cells s.c. and treated i.p. when tumors reached $20-30 \mathrm{~mm}^{2}$ in size with $10 \mathrm{mg} / \mathrm{kg}$ OXA or vehicle (PBS). Mice of the same age and gender were randomly allocated in the different experimental groups, ensuring an equal distribution of tumor sizes for therapeutic experiments.

\section{Gut colonization with dedicated species}

The tested species, Bacteroides fragilis and Paraprevotella clara, were isolated by culturomics from ileum samples from a previous study [5]. Species were grown on 5\% sheep blood-enriched Columbia agar (bioMerieux) in an anaerobic atmosphere created using anaerobic generators (bioMerieux) at $37^{\circ} \mathrm{C}$ for $24-72 \mathrm{~h}$. Colonization of MC38-bearing SPF mice was performed by oral gavage with each species ( $n=6$ mice per group). For bacterial gavage, colonies were resuspended in PBS at a concentration of $10^{9} \mathrm{CFU} / \mathrm{mL}$, evaluated using a fluorescence spectrophotometer (Eppendorf) at an optical density of 1 measured at a wavelength of $600 \mathrm{~nm}$. Two bacterial gavages were performed for each mouse, the first, $24 \mathrm{~h}$ before the treatment with OXA and then $24 \mathrm{~h}$ after the treatment.

\section{Flow cytometry analyses}

Tumors and ileal cells were harvested at the end of the experiment for immunological analysis. Gut dissociation to harvest cells from the epithelial compartment was performed as previously described [38]. Briefly, ilea and/or colons were collected and fat tissue, Peyer's patches, and feces were removed. Intestines were cut longitudinally and then cut transversally into small pieces into a tube. Pieces were transferred into a new $50-\mathrm{mL}$ tube with $20 \mathrm{~mL}$ of dissociation medium (PBS, 5\% FCS, 5 mM EDTA, and 1 $\mathrm{mM}$ DTT), vortexed, and shaken at $37^{\circ} \mathrm{C}$ for $15 \mathrm{~min}$. Cell suspensions were collected in a new tube, filtered with a cell strainer $(100 \mu \mathrm{m})$, centrifuged, and resuspended in PBS. The dissociation steps were performed twice and cell suspensions stored on ice until use. For tumor dissociation, tumors were cut into pieces of 2-mm side in RPMI medium, digested with Liberase ${ }^{\mathrm{TM}} \mathrm{TM}$ (Sigma) and DNAse I (Roche) for $30 \mathrm{~min}$ at $37^{\circ} \mathrm{C}$. Subsequently, the cells were meshed through a $100-\mu \mathrm{m}$ cell strainer to retrieve singlecell suspensions. In all cases, two million cells were preincubated with purified anti-mouse CD16/CD32 (eBioscience, catalog reference 16-0161-86) for 20 min at 4 ${ }^{\circ} \mathrm{C}$, before membrane staining. Dead cells were excluded using the Live/Dead Fixable Yellow dead cell stain kit (Life Technologies). For cell phenotyping, the following antibodies were purchased from BD Pharmingen: anti-mouse mouse CD45 (catalog reference: 560510), anti-mouse CXCR5 (catalog reference: 551960), and anti-mouse CD3e (catalog reference: 552774). The following antibodies were purchased from BioLegend: anti-mouse CD4 (catalog reference: 100428), anti-mouse CXCR3 (catalog reference: 126531). The following antibodies were purchased from $R \& D$ systems: anti-mouse CCR6 (catalog reference: FAB590P). The following antibody was purchased from Invitrogen: anti-mouse PD-1 (catalog reference: 17-9985-82). Samples were acquired on 13-color Cytoflex (Beckman Coulter) and analyses were performed with FlowJo v10 software (Tree Star, Ashland, OR, USA).

\section{External data availability of 16S RNA seq}

Microbiota characterization was already reported [5]. All raw sequencing data can be found at the NCBI Sequence Read Archive (accession number: PRJNA478491; "Ileal Apoptosis and Microbiome Shape Immunosurveillance and Prognosis of Proximal Colon Cancer", https://www.ncbi.nlm.nih.gov/ bioproject/?term=PRJNA478491, or https://www.ncbi.nlm. nih.gov/sra/PRJNA478491).

\section{Statistical analysis}

The Mann-Whitney $U$ test was used in comparisons of two group parameters, after Kruskal-Wallis test for multiple groups. The false-discovery rate was tested by the Benjamini-Hochberg procedure [39]. Survival curves were estimated using the Kaplan-Meier product limit method. Association between variables and outcome was evaluated using the log-rank test. Hierarchical clustering has been done with the distance 1-Pearson correlation coefficient and Ward's agglomeration method. Statistics and graphics were performed using the R software and GraphPad Prism v7.03. All tests were two-sided, with mean \pm SEM depicted, and $P$ values $<0.05$ were considered statistically significant.

For human sample studies, the investigators were blinded to group allocation during data collection and analysis. For mice experiments, the investigators were not blinded to group allocation when performing treatments during the experiment, but they were blinded during data collection of outcome and analysis.

Acknowledgements We are thankful to the animal facility team of GRCC and VIB U-Ghent. We are thankful to Hannah Vikula from VIB U-Ghent for technical assistance. We are thankful to technicians and pathologists from Centre GF Leclerc and the HistoCytoPathologie (HCP) module of the Translational Research Laboratory at GRCC. Service de Chirurgie Digestive et Oncologique, Hôpital Bicêtre, Le Kremlin-Bicêtre, France. LZ and GK were supported by the Ligue 
contre le Cancer (équipe labelisée); Agence Nationale de la Recherche (ANR) francogermanique ANR-19-CE15-0029, ANR Projets blancs; ANR under the frame of E-Rare-2, the ERA-Net for Research on Rare Diseases; Association pour la recherche sur le cancer (ARC); BMS Foundation, Cancéropôle Ile-de-France; Chancellerie des Universités de Paris (Legs Poix), Fondation pour la Recherche Médicale (FRM); a donation by Elior; the European Commission (ArtForce); the European Research Council (ERC); Institut National du Cancer (INCa); Inserm (HTE); Institut Universitaire de France; LeDucq Foundation; the LabEx Immuno-Oncology; the RHU Torino Lumière (ANR-16RHUS-0008); H2020 ONCOBIOME; the SIRIC Stratified Oncology Cell DNA Repair and Tumor Immune Elimination (SOCRATE); the SIRIC Cancer Research and Personalized Medicine (CARPEM); FHU CARE, The Dassault Group. M.P.R. was supported by a philanthropist funding by Elisabeth Badinter.

Author contributions Conceptualization: MPR and LZ; Performing experiments and data collection: MPR, MP, SY, KS, IP, MTA, C. Rauber, BR, SB, PL, EP, CHKL, LA, and PO; Formal data analysis: C. Richard, CK, and VI; Data curation, VI; Reagents, animal models, and clinical sample resources: DD, JYS, AH, DM, FG, GB, LV, and GVL.; Writing-Original draft, MPR; Writing-Review and editing, MPR and LZ; Visualization, MP; Supervision, GK and LZ; Funding acquisition, MPR, IGB, GK, and LZ.

\section{Compliance with ethical standards}

Conflict of interest LZ and GK are cofounders of EverImmune, a biotech company devoted to the use of commensal bacteria for the treatment of cancers. MPR and LZ hold patent PCT/EP2018/079878 related to treatments and gut prognostic signatures in colorectal cancer based on these results.

Publisher's note Springer Nature remains neutral with regard to jurisdictional claims in published maps and institutional affiliations.

Open Access This article is licensed under a Creative Commons Attribution 4.0 International License, which permits use, sharing, adaptation, distribution and reproduction in any medium or format, as long as you give appropriate credit to the original author(s) and the source, provide a link to the Creative Commons license, and indicate if changes were made. The images or other third party material in this article are included in the article's Creative Commons license, unless indicated otherwise in a credit line to the material. If material is not included in the article's Creative Commons license and your intended use is not permitted by statutory regulation or exceeds the permitted use, you will need to obtain permission directly from the copyright holder. To view a copy of this license, visit http://creativecommons. org/licenses/by/4.0/.

\section{References}

1. Le DT, Uram JN, Wang H, Bartlett BR, Kemberling H, Eyring $\mathrm{AD}$, et al. PD-1 blockade in tumors with mismatch-repair deficiency. N Engl J Med. 2015;372:2509-20.

2. Van Cutsem E, Cervantes A, Adam R, Sobrero A, Van Krieken JH, Aderka D, et al. ESMO consensus guidelines for the management of patients with metastatic colorectal cancer. Ann Oncol. 2016;27:1386-422.

3. Zitvogel L, Ma Y, Raoult D, Kroemer G, Gajewski TF. The microbiome in cancer immunotherapy: Diagnostic tools and therapeutic strategies. Science. 2018;359:1366-70.
4. Maynard CL, Elson CO, Hatton RD, Weaver CT. Reciprocal interactions of the intestinal microbiota and immune system. Nature. 2012;489:231-41.

5. Roberti MP, Yonekura S, Duong CPM, Picard M, Ferrere G, Tidjani Alou M, et al. Chemotherapy-induced ileal crypt apoptosis and the ileal microbiome shape immunosurveillance and prognosis of proximal colon cancer. Nat Med. 2020;26:919-31.

6. Bindea G, Mlecnik B, Tosolini M, Kirilovsky A, Waldner M, Obenauf AC, et al. Spatiotemporal dynamics of intratumoral immune cells reveal the immune landscape in human cancer. Immunity. 2013; 39:782-95.

7. Galon J, Fridman W-H, Pagès F. The adaptive immunologic microenvironment in colorectal cancer: a novel perspective: Fig. 1. Cancer Res. 2007;67:1883-6.

8. Galon J, Angell HK, Bedognetti D, Marincola FM. The continuum of cancer immunosurveillance: prognostic, predictive and mechanistic signatures. Immunity. 2013;39:11-26.

9. Pagès F, Mlecnik B, Marliot F, Bindea G, Ou F-S, Bifulco C, et al. International validation of the consensus immunoscore for the classification of colon cancer: a prognostic and accuracy study. Lancet. 2018;391:2128-39.

10. Slowicka K, Petta I, Blancke G, Hoste E, Dumas E, Sze M, et al. Zeb2 drives invasive and microbiota-dependent colon carcinoma. Nat Cancer. 2020;1:620-34.

11. Irrazábal T, Belcheva A, Girardin SE, Martin A, Philpott DJ. The multifaceted role of the intestinal microbiota in colon cancer. Mol Cell. 2014;54:309-20.

12. Geng J, Fan H, Tang X, Zhai H, Zhang Z. Diversified pattern of the human colorectal cancer microbiome. Gut Pathog. 2013;5:2.

13. Sears CL, Garrett WS. Microbes, microbiota, and colon cancer. Cell Host Microbe. 2014;15:317-28.

14. Iida N, Dzutsev A, Stewart CA, Smith L, Bouladoux N, Weingarten RA, et al. Commensal bacteria control cancer response to therapy by modulating the tumor microenvironment. Science. 2013;342:967-70.

15. Kryczek I, Wu K, Zhao E, Wei S, Vatan L, Szeliga W, et al. IL-17 + regulatory $\mathrm{T}$ cells in the microenvironments of chronic inflammation and cancer. J Immunol. 2011;186:4388-95.

16. Kimura A, Naka T, Nohara K, Fujii-Kuriyama Y, Kishimoto T. Aryl hydrocarbon receptor regulates Stat 1 activation and participates in the development of Th17 cells. Proc Natl Acad Sci USA. 2008;105:9721-6.

17. Johansson-Lindbom B, Agace WW. Generation of gut-homing $\mathrm{T}$ cells and their localization to the small intestinal mucosa. Immunol Rev. 2007;215:226-42.

18. Peske JD, Woods AB, Engelhard VH. Control of CD8 T-cell infiltration into tumors by vasculature and microenvironment. Adv. Cancer Res. 2015;128:263-307.

19. Jacquelot N, Enot DP, Flament C, Vimond N, Blattner C, Pitt JM, et al. Chemokine receptor patterns in lymphocytes mirror metastatic spreading in melanoma. J Clin Investig. 2016;126:921-37.

20. Routy B, Le Chatelier E, Derosa L, Duong CPM, Alou MT, Daillère $\mathrm{R}$, et al. Gut microbiome influences efficacy of PD1-based immunotherapy against epithelial tumors. Science. 2018; 359:91-97.

21. Li Y, Tinoco R, Elmén L, Segota I, Xian Y, Fujita Y, et al. Gut microbiota dependent anti-tumor immunity restricts melanoma growth in Rnf5-/- mice. Nat Commun. 2019;10:1492.

22. Jang MH, Sougawa N, Tanaka T, Hirata T, Hiroi T, Tohya K, et al. CCR7 is critically important for migration of dendritic cells in intestinal lamina propria to mesenteric lymph nodes. J Immunol. 2006;176:803-10.

23. Cremonesi E, Governa V, Garzon JFG, Mele V, Amicarella F, Muraro MG, et al. Gut microbiota modulate T cell trafficking into human colorectal cancer. Gut. 2018;67:1984-94. 
24. Ma C, Han M, Heinrich B, Fu Q, Zhang Q, Sandhu M, et al. Gut microbiome-mediated bile acid metabolism regulates liver cancer via NKT cells. Science. 2018;360:eaan5931.

25. Loo TM, Kamachi F, Watanabe Y, Yoshimoto S, Kanda H, Arai $\mathrm{Y}$, et al. Gut microbiota promotes obesity-associated liver cancer through PGE 2-mediated suppression of antitumor immunity. Cancer Discov. 2017;7:522-38.

26. Tanoue T, Morita S, Plichta DR, Skelly AN, Suda W, Sugiura Y, et al. A defined commensal consortium elicits CD8 T cells and anti-cancer immunity. Nature. 2019;565:600-5.

27. Wang L, Tang L, Feng Y, Zhao S, Han M, Zhang C et al. A purified membrane protein from Akkermansia muciniphila or the pasteurised bacterium blunts colitis associated tumourigenesis by modulation of CD8 ${ }^{+} \mathrm{T}$ cells in mice. Gut. 2020;69: 1988-97.

28. Fluckiger A, Daillère R, Sassi M, Sixt BS, Liu P, Loos F, et al. Cross-reactivity between tumor MHC class I-restricted antigens and an enterococcal bacteriophage. Science. 2020;369:936-42.

29. Gil-Cruz C, Perez-Shibayama C, De Martin A, Ronchi F, van der Borght K, Niederer R, et al. Microbiota-derived peptide mimics drive lethal inflammatory cardiomyopathy. Science. 2019;366:881-6.

30. Parhi L, Alon-Maimon T, Sol A, Nejman D, Shhadeh A, FainsodLevi $\mathrm{T}$, et al. Breast cancer colonization by Fusobacterium nucleatum accelerates tumor growth and metastatic progression. Nat Commun. 2020;11:3259.

31. Nejman D, Livyatan I, Fuks G, Gavert N, Zwang Y, Geller LT, et al. The human tumor microbiome is composed of tumor type-specific intracellular bacteria. Science. 2020;368:973-80.

32. Benedix F, Schmidt U, Mroczkowski P, Gastinger I, Lippert H, Kube R. Colon carcinoma - Classification into right and left sided cancer or according to colonic subsite? - Analysis of 29568 patients. Eur J Surgical Oncol. 2011;37:134-9.

33. Boeckx N, Koukakis R, Op de Beeck K, Rolfo C, Van Camp G, Siena $\mathrm{S}$, et al. Primary tumor sidedness has an impact on prognosis and treatment outcome in metastatic colorectal cancer: results from two randomized first-line panitumumab studies. Ann Oncol. 2017; 28:1862-8.

34. Arnold D, Lueza B, Douillard J-Y, Peeters M, Lenz H-J, Venook A, et al. Prognostic and predictive value of primary tumour side in patients with RAS wild-type metastatic colorectal cancer treated with chemotherapy and EGFR directed antibodies in six randomized trials. Ann Oncol. 2017;28: 1713-29.

35. Tumeh PC, Harview CL, Yearley JH, Shintaku IP, Taylor EJM, Robert L, et al. PD-1 blockade induces responses by inhibiting adaptive immune resistance. Nature. 2014;515:568-71.

36. Van Allen EM, Miao D, Schilling B, Shukla SA, Blank C, Zimmer L, et al. Genomic correlates of response to CTLA-4 blockade in metastatic melanoma. Science 2015;350: 207-11.

37. Bankhead P, Loughrey MB, Fernández JA, Dombrowski Y, McArt DG, Dunne PD, et al. QuPath: Open source software for digital pathology image analysis. Sci Rep. 2017;7:16878.

38. Sato T, Vries RG, Snippert HJ, van de Wetering M, Barker N, Stange DE, et al. Single Lgr5 stem cells build crypt-villus structures in vitro without a mesenchymal niche. Nature. 2009; 459:262-5.

39. Benjamini Y, Hochberg Y. Controlling the false discovery rate: a practical and powerful approach to multiple testing. J R Stat Soc Ser B (Methodol). 1995;57:289-300.

\section{Affiliations}

\section{Marion Picard ${ }^{1,2,3,4}$ - Satoru Yonekura $\mathbb{1}^{1,2,5} \cdot$ Karolina Slowicka $^{6,7} \cdot$ loanna Petta ${ }^{6,8} \cdot$ Conrad Rauber $^{1,2,5}$. Bertrand Routy ${ }^{1,2,5} \cdot$ Corentin Richard $^{9,10} \cdot$ Valerio lebba $^{11} \cdot$ Maryam Tidjani Alou $^{1,2} \cdot$ Sonia Becharef ${ }^{1,2} \cdot$ Pierre Ly $^{1,2}$. Eugenie Pizzato $^{1,2}$. Christian H. K. Lehmann ${ }^{12}$. Lukas Amon ${ }^{12}$. Christophe Klein $\mathbb{1}^{13} \cdot$ Paule Opolon $^{14}$. Ivo Gomperts Boneca $\mathbb{1}^{3,4,15}$. Jean-Yves Scoazec ${ }^{14}$ - Antoine Hollebecque ${ }^{16}$. David Malka ${ }^{16}$. François Ghiringhelli $\mathbb{1}^{9,10}$ - Diana Dudziak ${ }^{12}$ - Geert Berx ${ }^{7,17}$ - Lars Vereecke $\mathbb{1}^{6,8}$. Geert van Loo $\mathbb{1}^{6,7}$. Guido Kroemer ${ }^{13,18,19,20,21} \cdot$ Laurence Zitvogel $\mathbb{D}^{1,2,5,21,22} \cdot$ Maria Paula Roberti ${ }^{1,2,22,23}$}

1 Gustave Roussy Cancer Campus (GRCC), Villejuif, France

2 Institut National de la Santé Et de la Recherche Médicale (INSERM) U1015, Equipe Labellisée-Ligue Nationale contre le Cancer, Villejuif, France

3 Institut Pasteur, Unit Biology and Genetics of the Bacterial Cell Wall, Paris, France

4 INSERM, Equipe Avenir, Paris, France

5 Université Paris-Saclay, Le Kremlin-Bicêtre cedex, France

6 VIB Center for Inflammation Research, Ghent, Belgium

7 Department of Biomedical Molecular Biology, Ghent University, Ghent, Belgium

8 Department of Internal Medicine and Pediatrics, Ghent University, Ghent, Belgium

9 Department of Medical Oncology, Center GF Leclerc, Dijon, France
10 Plateform Transfer in Biological Oncology, Dijon, France

11 Department of Medical, Surgical and Health Sciences, University of Trieste, Trieste, Italy

12 Department of Dermatology, Laboratory of Dendritic Cell Biology, Medical Immunology Campus Erlangen, University Hospital of Erlangen, Friedrich-Alexander-University (FAU) of Erlangen-Nürnberg, Erlangen, Germany

13 Equipe Labellisée par la Ligue Contre le Cancer, Université de Paris, Sorbonne Université, INSERM U1138, Centre de Recherche des Cordeliers, Paris, France

14 Departement de Biologie et Pathologie Médicales, Gustave Roussy Cancer Campus, Villejuif, France

15 CNRS UMR2001, Paris, France

16 Departement de Médicine Oncologique, Gustave Roussy Cancer Campus, Villejuif, France 
17 Cancer Research Institute Ghent (CRIG), Ghent, Belgium

18 Cell Biology and Metabolomics Platforms, Gustave Roussy Cancer Campus, Villejuif, France

19 Pôle de Biologie, Hôpital Européen Georges Pompidou, Assistance Publique-Hôpitaux de, Paris, France

20 Department of Women's and Children's Health, Karolinska University Hospital, Stockholm, Sweden
21 Suzhou Institute for Systems Medicine, Chinese Academy of Medical Sciences, Suzhou, China

22 Center of Clinical Investigations in Biotherapies of Cancer (CICBT) 1428, Villejuif, France

23 Present address: Clinical Cooperation Unit Applied Tumor Immunity, National Center for Tumor Diseases (NCT), German Cancer Research Center (DKFZ), Heidelberg, Germany 